\title{
Phenomenology of pulsar B0809+74's rotating subbeam system
}

\section{Geometry and profile "absorption"}

\author{
Joanna M. Rankin ${ }^{1, \star}$, R. Ramachandran ${ }^{2}$, and Svetlana A. Suleymanova ${ }^{3,4}$ \\ 1 Sterrenkundig Instituut "Anton Pannekoek", 1098 SJ Amsterdam, The Netherlands \\ e-mail: jrankin@astro.uva.nl, joanna.rankin@uvm.edu \\ 2 Department of Astronomy, University of California, Berkeley, CA 94720, USA \\ e-mail: ramach@astron. berkeley.edu \\ 3 Pushchino Radio Astronomy Observatory, 142290 Pushchino, Russia \\ e-mail: suleym@prao.psn.ru \\ ${ }^{4}$ Isaac Newton Institute of Chile, Pushchino Branch
}

Received 3 November 2004 / Accepted 23 August 2005

\section{ABSTRACT}

The basic emission geometry of pulsar B0809+74 is modeled after determinating its polarization position-angle traverse. As the star's profiles are truncated by "absorption" over a broad band, most severely between some 60 and $1500 \mathrm{MHz}$, the analysis also provides quantitative estimates of the extent of this effect at each frequency. The model's predicted scales are found to agree closely with those determined by correlating pulse sequences observed simultaneously in different bands and by measuring $P_{2}$ at different frequencies.

Key words. stars: pulsars: individual: B0809+74 - polarisation - radiation mechanisms: non-thermal

\section{Introduction}

PSR B0809+74 ranks among the dozen most influential radio pulsars in the sky-and still provides one of the very richest contexts for studying their emission physics. It exhibits all six of the heretofore well identified phenomena: subpulse drifting, pulse nulling, profile mode switching, polarization modes, microstructure, and "absorption". Though not among the four original pulsars (Hewish et al. 1968; Pilkington 1968), it was discovered at Cambridge in their last "batch" (Cole \& Pilkington 1968), which also included B0329+54. Vitkevich \& Shitov (1970) first exhibited the star's $11-P_{1}$ drifting subpulses, and Taylor \& Huguenin (1971) then demonstrated their remarkable stability via a fluctuation spectrum, while also showing that it occasionally "ceased pulsing" for a few periods.

Another crucial characteristic of B0809+74 was first noted by Page (1973), and then well documented via attempts to reconcile simultaneous pulse-sequence (hereafter PS) observations made with the Pushchino (hereafter PRAO) array at 102.5 MHz and the Effelsberg telescope (hereafter MPIfR) at $1.72 \mathrm{GHz}$, whereupon it became clear from longitudelongitude correlation maps that a leading portion of the higher frequency pulse corresponded to no part of the pulse at meter wavelengths. This finding that a substantial leading part of the lower frequency profile was "missing" - and indeed over

^ On leave from Physics Dept., University of Vermont, Burlington, VT 05405, USA. a broad band extending at least between $100 \mathrm{MHz}$ and $1 \mathrm{GHz}$ - Bartel et al. (also Bartel, both 1981) argued that the missing portion was absorbed. This perplexing phenomenon has also been well investigated (Davies et al. 1984; Popov 1987; Popov \& Sieber 1990; see also Rankin 1983), and while the physical origin of the effect is still not understood, B0809+74 remains the defining example of this "absorption" phenomenon.

Similarly, the star also exhibits the curious and virtually unique phenomenon that sets of well measured, broad band, time-aligned profiles cannot readily be reconciled with any nominal value of the dispersion measure $(D M)$. While a part of the effect can be seen in Davies et al. (their Fig. 2), this strange behavior naturally led to speculation that the star's magnetic field is distorted by non-dipolar contributions (see also Popov 1987). Some other measurements and interpretation prompted discussions of "superdispersion" (e.g., Shitov \& Malofeev 1985; Izvekova et al. 1990); whereas followup observations at $25 \mathrm{MHz}$ and below using the Kharkov array (Bruk et al. 1986) found only a dispersive behavior, and recent work by Kuz'min and his colleagues at frequencies up to $10 \mathrm{GHz}$ suggest progressive non-dispersive offsets above $21 \mathrm{~cm}$ (1420 MHz) (Kuz'min et al. 1985, 1986, 1998). One very welcome clarification amid these complex findings has been the identification of microstructure in the star's PSs at very low frequencies (Soglasnov et al. 1981; Novikov et al. 1984) and its use to accurately and independently determine the star's DM through cross-correlation of well measured PSs at adjacent frequencies below $100 \mathrm{MHz}$. This resulting value is 
$5.7513 \pm 0.0002 \mathrm{pc} \mathrm{cm}^{-3}$ as measured by Popov et al. (1987) using PRAO observations at 102.5, 81.2 and 69.7 MHz.

Paradoxically, the role of the polarization modes was not fully studied in B0809+74 until recently, because the early polarimetric studies of this pulsar (Taylor et al. 1971; Lyne et al. 1971) were carried out before this phenomenon was well identified (Manchester et al. 1975; Backer \& Rankin 1980). Ramachandran et al. (2002) have now demonstrated their determinative role in the star's PS polarization, whereas other recent polarimetric studies (e.g., von Hoensbroech \& Xilouris 1997; Gould \& Lyne 1998) clearly reconfirm the remarkable complexity of the modal depolarization effects of its profiles. It is now clear that the star's largely complete profile depolarization at meter wavelengths is typical of the $\left(\boldsymbol{S}_{\mathrm{d}}\right)$ pulsar class (Rankin \& Ramachandran 2003) where the sightline runs tangentially along the outside conal edge. Both the linear depolarization and slightly nonorthogonal polarization-mode mixing in this outside conal edge region of the emission beam tend to distort the overall average polarization-angle (hereafter PA) traverse (Ramachandran et al. 2002; e.g., Ramachandran et al. 2004, for a similar effect in B2016+28) and frustrate any ready geometrical interpretation for such stars (e.g., Lyne \& Manchester 1988; Rankin 1993a,b). It has been possible to show that the complex polarization-modal structure of the drifting subpulses in B0809+74 are largely responsible for the old and perplexing reports of both longitude and frequency variations in the subpulse interval $P_{2}$ (Rankin et al. 2005, hereafter RRS; see also Edwards \& Stappers 2003, 2004).

In view of all these circumstances, pulsar B0809+74 undoubtedly provides the currently most important and potentially insightful context for further analysis using the techniques developed by Deshpande \& Rankin $(1999,2001)$ for B0943+10. We have found their application to this star, however, quite challenging. Here, we take up the issue of the star's notorious "absorption" in an effort to understand its underlying emission geometry. In Sect. 2 we describe the observations on which our analyses below are based, and Sect. 3 gives a discussion of the star's modal polarization-angle traverse. Section 4 outlines the "absorption", Sect. 5 assembles a geometric model, and we discuss our results in Sect. 6.

\section{Observations}

The primary Westerbork Synthesis Radio Telescope (hereafter WSRT) observations at 1380 and $328 \mathrm{MHz}$ and Pushchino Radio Astronomy Observatory (PRAO) sequences at 112.7 and 41.0 $\mathrm{MHz}$ used in our analyses below are identical to those studied previously (and described in detail) by Ramachandran et al. (2002) and Rankin et al. (2005); therefore, we here merely summarize their main properties in Table 1 . In addition we will make use of a set of $328,382,1375$, and $4880 \mathrm{MHz}$ WSRT total-power average profiles to study their relative alignment. Note that while the bright 2000 November 26 observation at $328 \mathrm{MHz}$ is the same one first studied by Ramachandran et al. (2002), it has now been corrected for a recently determined instrumental effect that converted some linear into circular polarization (Edwards \& Stappers 2004; see their Appendix).
Table 1. Pulse-sequence polarimetry observations.

\begin{tabular}{cccc}
\hline \hline $\begin{array}{c}\text { Frequency } \\
(\mathrm{MHz})\end{array}$ & Date & $\begin{array}{c}\text { BW/chans } \\
(\mathrm{MHz} / \#)\end{array}$ & $\begin{array}{c}\text { Resolution } \\
\left({ }^{\circ}\right)\end{array}$ \\
\hline 4880 & 2001 May 30 & $80 / 128$ & 0.114 \\
1375 & 2001 May 30 & $80 / 256$ & 0.114 \\
1380 & 2002 Jan 10 & $80 / 256$ & 0.114 \\
382 & 2001 May 30 & $10 / 256$ & 0.114 \\
328 & 2000 Nov. 26 & $10 / 256$ & 0.114 \\
112.7 & 2000 Feb. 06 & $2.56 / 128$ & 0.71 \\
41.0 & 2003 Dec. 27 & $0.16 / 128$ & 1.43 \\
\hline
\end{tabular}

\section{Defining the PA sweep rate}

While many pulsars with conal single $\left(\boldsymbol{S}_{\mathrm{d}}\right)$ profiles have substantially depolarized profiles and poorly defined PA traverses, this situation is particularly difficult for B0809+74. One need only inspect the well measured average polarization profiles of Gould \& Lyne (1998) and then compare them with the equally well observed high frequency profiles of von Hoensbroech \& Xilouris (1997) to see that no consistent value for the PA-sweep rate can be determined for this pulsar from polarized profile measurements. This circumstance is also well exemplified at our frequency of $328 \mathrm{MHz}$, where the aggregate polarization is hardly $10 \%$ - though we have just seen above that the fractional linear polarization of the individual pulses is quite consistently some $60 \%$ - and the average PA wanders through a traverse of some $100^{\circ}$ in a manner unrecognizable in terms of the single-vector (Radhakrishnan \& Cooke 1969; Komesaroff 1970) model. Ramachandran et al. (2002) were the first to measure the pulsar's individual-pulse polarization with sufficient resolution and precision to distinguish the two modal trajectories, and the PA density diagram given as their Fig. 1 shows two modal PA "tracks" that overlap over most of the width of the profile and thus together largely depolarize it.

The lower panel of Fig. 1 is similar to the above Fig. 1, giving the average polarized profile and the PA density, but here our analysis has had the benefit of the Westerbork polarimetry calibration described in Edwards \& Stappers' (2004) Appendix. Let us inspect this display critically as we consider how to quantify the PA-traverse information it displays. It comes as no surprise either that the PAs assume two well delineated modal "tracks", or that these two PA distributions are prominent over different ranges of longitude - we can also see this behavior clearly in the PS polarization display of Ramachandran et al.'s (2002) Fig. $4^{1}$. More perplexing is the suggestion that the two "tracks" represent different PA sweep rates - though this impression is hard to gauge accurately given the complex manner in which the signal-to-noise ratio (hereafter $\mathrm{S} / \mathrm{N}$ ) of $L$ varies as a function of PA and longitude.

The lower panel of Fig. 1 then illustrates our method for fitting the two PA "tracks". First, a region is defined in which only one of the two modes is active. This is indicated by the dotted boundaries. Within this region, we have carried out a

\footnotetext{
The circular polarization in this display should be discounted, and the fractional linear taken as being about $50 \%$ greater per the recent WSRT recalibration by Edwards \& Stappers (2004). The configurations of modal polarization, however, remain nearly unaltered.
} 


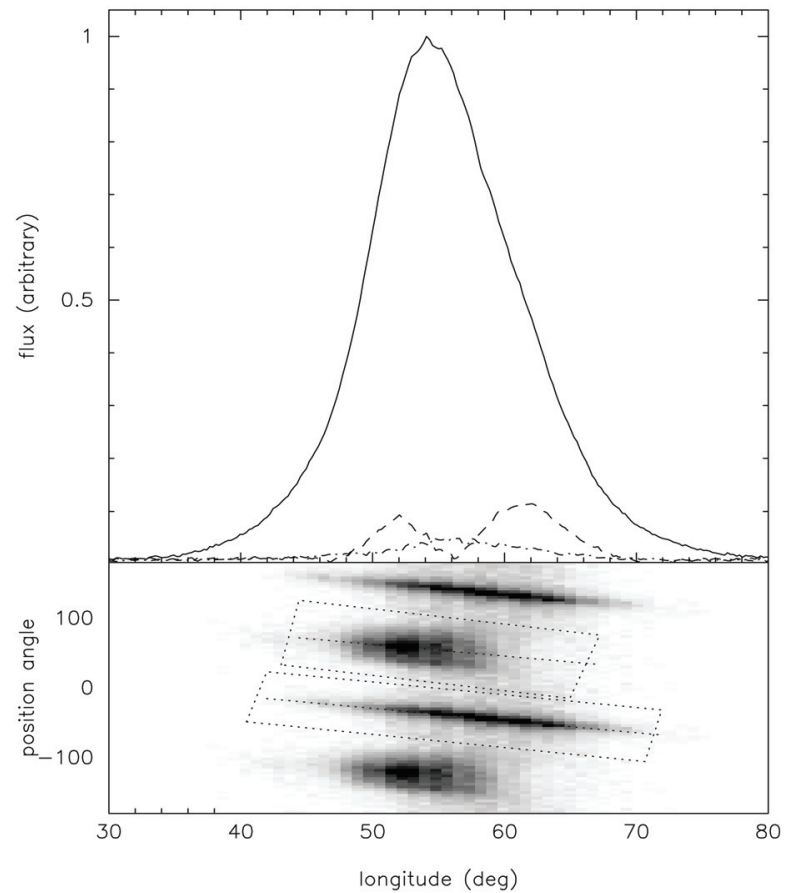

Fig. 1. PA-density diagram for pulsar B0809+74 at $328 \mathrm{MHz}$ after Ramachandran et al. (bottom panel; shown twice). The polarized profile (total power $I$ (solid curve), linear polarization $L$ (dashed), and circular polarization $V$ (dash-dotted)) is plotted in the top panel. The bottom panel by contrast depicts our procedure for fitting linear curves to the PA traverse: first a section of the PA distribution is taken which contains all significant power for a given mode (shown here by a dotted boundary), and these PA values are fitted using the linear power in each pixel as a weight (see text). The results, $-1.51 \pm 0.17$ and $-1.83 \pm 0.11^{\circ}{ }^{\circ}$ for the upper and lower modal boxes, respectively, were essentially independent of the bounding quadrangle as long as it was drawn to enclose the dark region of significant linearly polarized power.

weighted linear fit for the PA as a function of longitude, where the weights are computed as $L / \sigma$ within each PA-longitude "pixel" - that is, as the ratio of the linearly polarized power to the rms noise power. The results of the two fits do indeed differ in PA rate: the PA rate $R(=\Delta \chi / \Delta \varphi)$ for the upper modal box is $-1.51 \pm 0.17$ and $-1.83 \pm 0.11$ for the lower as indicated in the diagram.

It is difficult to understand how the two values of the PA sweep rate above could reflect different actual geometrical circumstances of modal emission. Moreover, a closer look at the two PA tracks shows that the steeper one has a sharply defined PA at each longitude and extends over nearly the entire profile width. The second track is shorter and suggestive at some points of a more complex PA distribution, but where best defined it falls nearly parallel to the steeper track. We therefore adopt the slope of the longer track as most indicative of the star's geometry.

\section{Attempting to understand B0809+74's "absorption"}

As mentioned above, the "absorption" identified over a broad meter-wavelength band in pulsar B0809+74's profile stands as the prototypical example of the phenomenon now so termed. While major efforts were made over more than a decade to determine whether multi-polar contributions to the star's magnetic field configuration or "superdispersion" might be physically responsible for the pulsar's outstanding behavior, we can now conclude that in the most basic observational terms "absorption" merely represents a failure of the star's profiles to exhibit the full widths, morphological evolution with frequency and consequent alignments expected for such an exemplar of the conal single $\left(\boldsymbol{S}_{\mathrm{d}}\right)$ class. Indeed, such effects are observed in most stars of this type (e.g., Rankin 1983), and a more dynamic conception of the phenomenon follows from analyses of the rotating-subbeam system of pulsar B0943+10 (Deshpande \& Rankin 2001; hereafter DR01).

Let us therefore look carefully at the profile evolution and alignment of B0809+74. Although the "absorption" was first identified by Bartel and collaborators in two 1981 papers, it was only three years later when Davies et al. published the first broadband set of time-aligned profiles. These spanned an interval between 29 and $1412 \mathrm{MHz}$ and were aligned with the then best determined $D M$ value of $5.762 \mathrm{pc} \mathrm{cm}^{-3}$, appearing as this paper's Fig. 2. The meter-wavelength profiles are "absorbed" here because their widths do not escalate with wavelength as expected - and, correlation studies of single pulses between 1720 and $102 \mathrm{MHz}$ clearly showed that while subpulses in the trailing part of the $18-\mathrm{cm}$ profile correlate well with $102-\mathrm{MHz}$ subpulses, those within the leading half of the $18-\mathrm{cm}$ profile have no counter part at $102 \mathrm{MHz}$ (Bartel et al. 1981). One might plausibly interpret the alignment of these profiles, then, as showing that their trailing edges roughly correspond and exhibit the expected outer cone monotonic increase in scale, whereas a leading-edge portion of the profiles in the band between about 900 and $90 \mathrm{MHz}$ is missing, so that the overall profile-width escalation is compromised. Note, however, that the profiles around $21 \mathrm{~cm}$ and below $60 \mathrm{MHz}$ appear to be more intact; here we see double profiles at the lower frequencies and correlations and other evidence which again suggest emission from both sides of the magnetic axis longitude at $21 \mathrm{~cm}$.

Over the next two years, further sets of time-aligned profiles were published by Kuz'min et al. (1985) and Bruk et al. (1986). We reproduce the first here as our Fig. $2^{2}$. While several of the profiles in Kuz'min et al. 's alignment are apparently the same as those in Davies et al. (e.g., those at 39, 62 and $92 \mathrm{MHz}$ ), the Kuz'min assemblage unaccountably omits the Pushchino 29-MHz observation while adding Effelsberg profiles at 4.6 and $10.7 \mathrm{GHz}$. This work also benefits from Popov et al. 's (1987) microstructure DM determination (prior to its publication), so that its low frequency profiles no longer tend to curl slightly to the left. At and below $21 \mathrm{~cm}$, the Davies et al. and Kuz'min et al. alignments are virtually identical, but Kuz'min's new 4.6- and 10.7-GHz profiles suggest a failure to align at very high frequency. Indeed, this circumstance was reiterated recently with new observations by Kuz'min et al. (1998), and in both cases the leading edges of the $10-\mathrm{GHz}$

\footnotetext{
2 This paper is a Lebedev Institute preprint of the manuscript which was eventually published as Kuz'min et al. (1986); however, the latter omits the figure in question.
} 


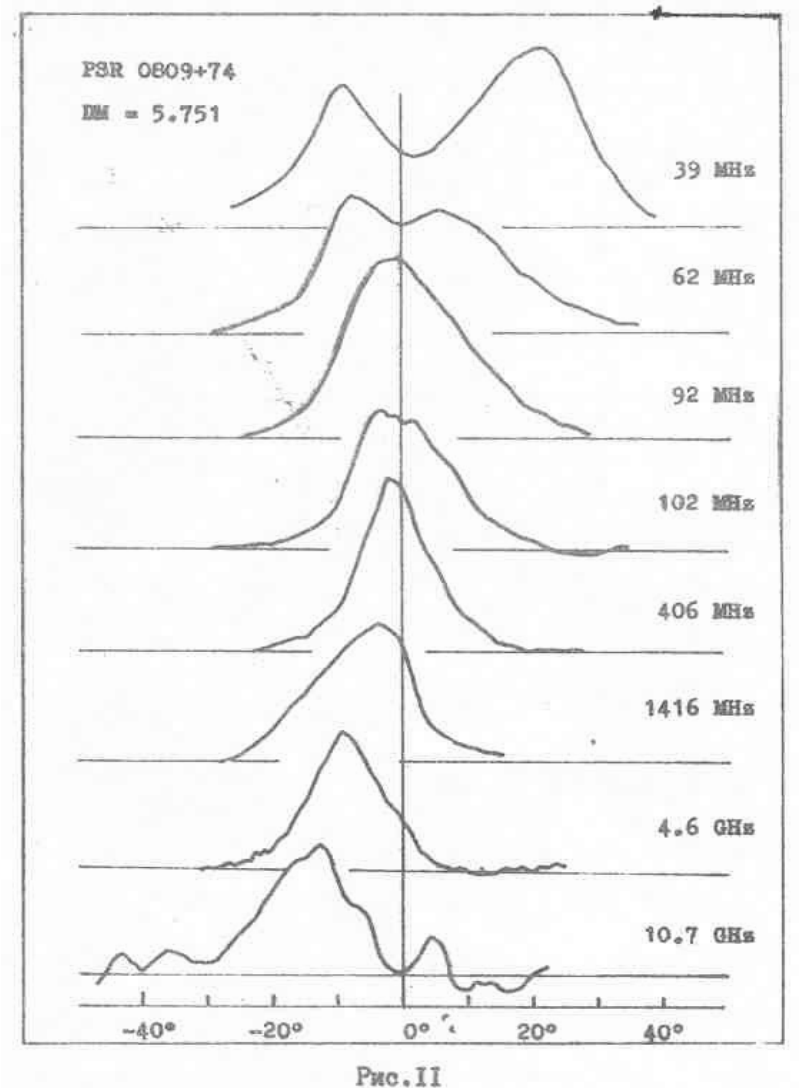

Fig. 2. Time-aligned profiles between $39 \mathrm{MHz}$ and $10.7 \mathrm{GHz}$, observed at PRAO, Jodrell Bank and Effelsberg, after Kuz'min et al. (1985). Several of the profiles are common to the similar time-aligned assemblage given by Davies et al. (1984), though the alignment here is carried out with Popov et al.'s (1987) then new microstructuredetermined $D M$ value of $5.7513 \mathrm{pc} \mathrm{cm}^{-3}$. At $21 \mathrm{cms}$ and below, the trailing edges of the profiles appear to correspond and exhibit the expected outer-cone increases in longitude scale (RFM). Similarly, the leading edges of the $21-\mathrm{cm}$ and $62-\mathrm{MHz}$ profiles may nearly correspond (as suggested by the recovering leading-edge correlation between $102 \mathrm{MHz}$ and $18 \mathrm{~cm}$ (Popov 1987)), but those at all intermediate frequencies seem to be missing a leading-edge portion of their profiles. It is just this circumstance which prompted Bartel and his collaborators in 1981 to suggest that the pulsar's leading-edge emission suffers "absorption". The 10-GHz profiles in both this set and in a much later compilation by Kuz'min et al. (1998) appear to fall well in advance of their lower frequency counterparts (see text) - suggesting a further anomaly in the alignment at very high frequencies.

profiles seem to fall some 2-3 degrees in advance of the leading edges of their $21-\mathrm{cm}$ counterparts. In addition, the $10-\mathrm{GHz}$ profiles are a little narrower than those at $21 \mathrm{~cm}$ and thus their trailing edges fall far in advance of those at lower frequencies.

The remarkable Bruk et al. (1986) work using the great UTR-2 array in Kharkov, Ukraine - which reports detections of the pulsar down to $12 \mathrm{MHz}$ and aligns a set of three well measured profiles at 17,20 and $25 \mathrm{MHz}$ - puts to rest the question of "superdispersion" as these very very low frequency profiles are found to align perfectly using a $D M$ of $5.752 \pm$ $0.005 \mathrm{pc} \mathrm{cm}^{-3}$. These observations also show that B0809+74's profile form changes little at very low frequencies; the double

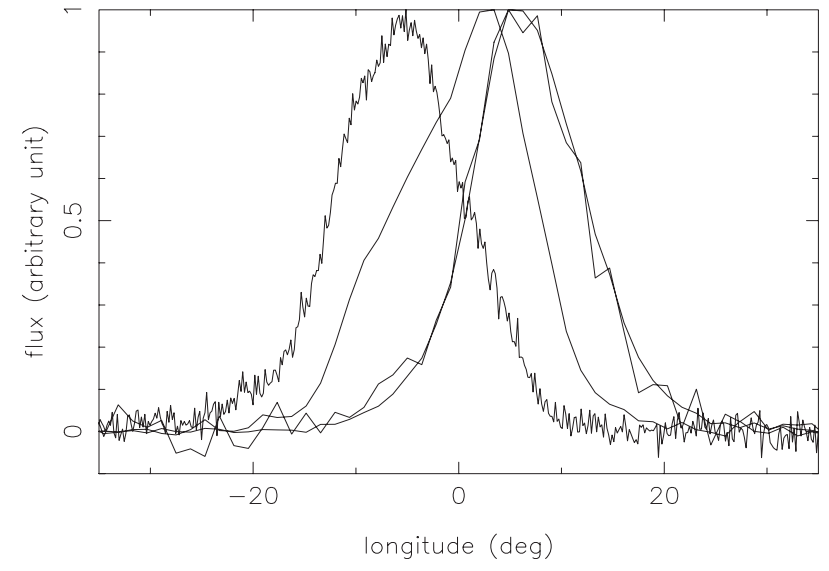

Fig. 3. A display of profiles at 382 (rightmost profiles), 1375 (center profile) and $4880 \mathrm{MHz}$ (lefthand profile) assembled to explore the high frequency alignment. Two sets of simultaneous observations at $1475 / 382$ and $4880 / 382 \mathrm{MHz}$, observed with the WSRT telescope, were used. The two $382-\mathrm{MHz}$ profiles were aligned by cross correlation as shown. The WSRT 5-GHz profile was of poor quality, so this was aligned by correlation with an EPN 4.85-GHz profile from Kijak et al. (1997), and it is this latter profile which is plotted. The relative positions of these profiles largely corroborate the alignments of Kuz'min et al. (1985). The longitude origin is taken at about the leading half-power point on the $382-\mathrm{MHz}$ profiles for reason explained later (see text).

form with the first component much weaker than the second seems to obtain throughout this entire band.

We have thus made an attempt to explore the character of the reported profile misalignment at very high frequency by aligning several different frequency WSRT profiles using Popov et al.'s $D M$ value of $5.7513 \mathrm{pc} \mathrm{cm}^{-3}$, and the results are depicted in Fig. 3. Pairs of simultaneous 4880/382- and $1375 / 382-\mathrm{MHz}$ observations were used, and the two lower frequency profiles then aligned by cross-correlation. Also, due to the poor $\mathrm{S} / \mathrm{N}$ of the WSRT $5-\mathrm{GHz}$ profile, it was used only to position a $4.85-\mathrm{GHz} \mathrm{EPN}^{3}$ profile contributed by Kijak et al. (1997), and this is what is depicted. Starting with the $21-\mathrm{cm}$ (1375-MHz) profile, which appears as expected to be the most complete, we confirm that the leading edge of the $5-\mathrm{GHz}$ profile leads the former by a good $3^{\circ}$, whereas the trailing edges of the $382-\mathrm{MHz}$ profiles lag by a similar amount.

While the evidence suggests that the $21-\mathrm{cm}$ profile is relatively complete, nothing at all confirms that it is fully so. The higher quality observations (e.g., von Hoensbroech \& Xilouris 1997; Gould \& Lyne 1998) show that it has an asymmetrial, trapezoidal shape from which a part of its leading edge could plausibly have been truncated. Indeed, we know that this is so from the foregoing polarimetric studies: the leading part of the $21-\mathrm{cm}$ profile is highly linearly polarized - a nearly unique circumstance among "drifters" in general, and as we have seen not the case at other frequencies even for B0809+74. This linear indicates that the subpulses in the leading half of profile at 21-cm are unimodal, and the missing mode is the one which at lower frequencies has the larger conal radius (RRS).

${ }^{3}$ European Pulsar Network: http://www .mpifr-bonn.mpg.de/ div/pulsar/data/ 


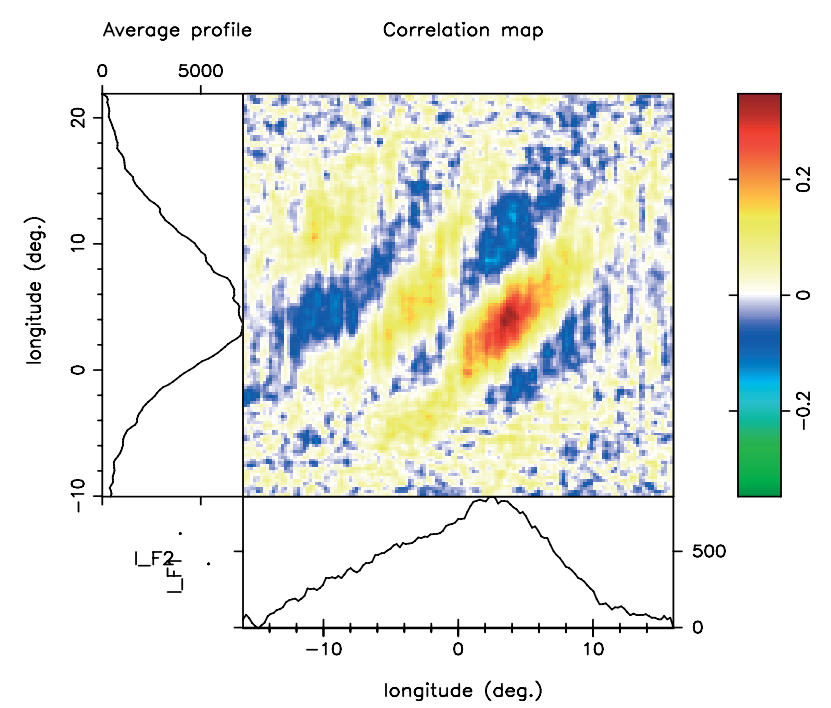

Fig. 4. Longitude-longitude correlation diagram at zero delay computed using the simultaneous $382 / 1375-\mathrm{MHz}$ PSs of 2001 May 30 recorded at the WSRT telescope. The higher and lower frequency profiles are plotted in the lower and lefthand panels, respectively. Note the restricted region of correlation which has a maximum well past $0^{\circ}$ longitude - that of the magnetic axis - in both cases. We also see that subpulses in the leading part of the $21-\mathrm{cm}$ profile exhibit only an indirect correlation (at intervals of $P_{2}$ ) with those at $382 \mathrm{MHz}$.

Thus, were it present, the $21-\mathrm{cm}$ profile would extend further in the leading direction, and, though it is difficult to estimate by just how much, the modal subbeams differ in peak radii by about $0.5-0.6^{\circ}$ (RRvLS) and this could produce a leading-edge longitude-width increase several times this on the outer cone edge. The much smaller fractional linear at $5 \mathrm{GHz}$ suggests that both polarization modes are again present here and thus its leading edge is intact ${ }^{4}$.

We should also speculate here about the trailing edge alignment between the $21-\mathrm{cm}$ and $382-\mathrm{MHz}$ profiles. In the next section we will see from the Thorsett-equation models that B0809+74's emission cone has a nearly constant radius in this frequency range, so the observed changes in profile width over the above interval are not primarily of geometric origin. Moreover, the 5-GHz profile's narrowness and misalignment suggests that the trailing-edge emission is falling off sharply, and it may well also have had some effect on the $21-\mathrm{cm}$ profile. Indeed, this is simply verified using the longitudelongitude correlation map of Fig. 4. The peaks of the $21-\mathrm{cm}$ and $382-\mathrm{MHz}$ profiles correlate most strongly at the same longitude (about $+4^{\circ}$ ) - again showing that the emission-cone radius is nearly constant between these two frequencies. Then note that the trailing-edge region of the $21-\mathrm{cm}$ profile is a few degrees shorter than its 382-MHz counterpart.

Davies et al. (1984) computed such a correlation plot from simultaneous observations made with Jodrell Bank at $406 \mathrm{MHz}$ and PRAO at $102 \mathrm{MHz}$ which appears as their Fig. 6. This

\footnotetext{
${ }^{4}$ The large fractional linear on B0809+74's leading edge is a very interesting phenomenon with potential physical implications. It occurs over a narrow band only between about 900 and $1400 \mathrm{MHz}$, and is substantially smaller at both $600 \mathrm{MHz}$ and $18 \mathrm{~cm}$.
}

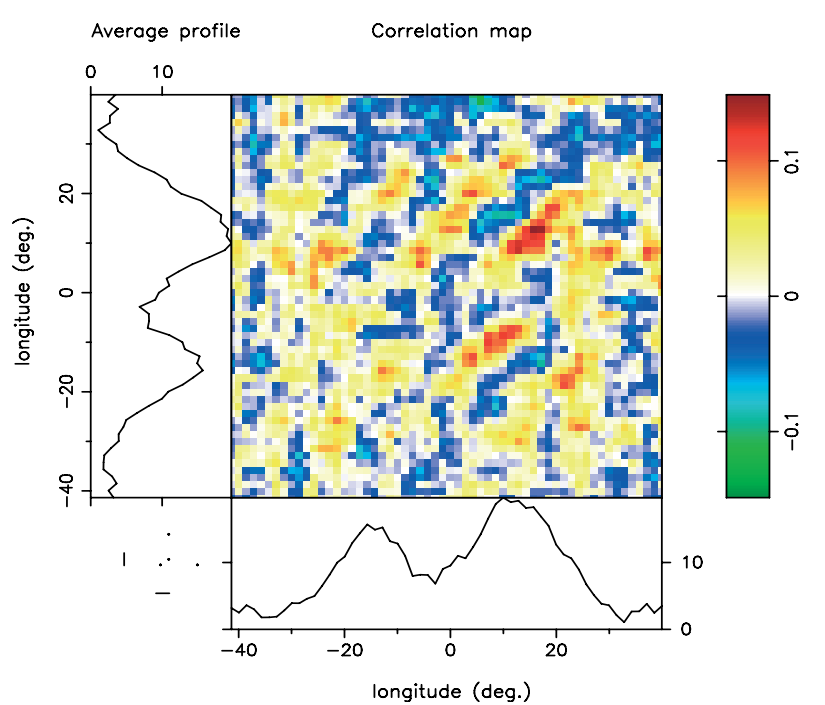

Fig. 5. 41-MHz longitude-longitude correlation diagram with a delay of one pulse for a 947-pulse PRAO PS acquired on 27 December 2003. The diagonal bands of positive and negative correlation are very evident but not very precisely defined. With careful measurements a $P_{2}$ value of $15-17^{\circ}$ can be obtained, which shows that the star's cone size has grown a little at this very low frequency.

plot very clearly shows a zone of significant correlation, whose peak associates the half-power point on the leading edge of the 406-MHz profile with a region near the corresponding $75 \%$-point of the $102-\mathrm{MHz}$ profile. The zone continues over nearly $30 \mathrm{~ms}\left(8^{\circ}\right)$ longitude. A handscale confirms that both profiles have equal scales and that the authors' sections through the correlation band has a slope of $1.4: 1$ as the text concludes. Further study, however, suggests that this slope is not well determined, and a slope of 1:1 cannot be ruled out. Also, it is perplexing that the correlation maximum occurs so far down the leading edge of the profile.

Popov (1986) computed another correlation diagram between an Effelsberg observation at $1.7 \mathrm{GHz}$ and a PRAO sequence at $102.5 \mathrm{MHz}$. His figure corroborates the earlier results of Bartel et al. whereby only emission at longitudes corresponding to the second half of the $1.7-\mathrm{GHz}$ profile correlate with the 102-MHz sequence - this strongly suggesting that a large early portion of the 102-MHz profile is "missing" or "absorbed". It also demonstrates that the correlation scale of the $102-\mathrm{MHz}$ sequence is extended with respect to the $21-\mathrm{cm}$ one. Popov associates the correlation maxima with the joint totalpower position of the pulsar's drifting subpulses, so his several parallel curves running along this oblique band are separated by an angular distance related to the subpulse spacing $P_{2}$, but we have seen in RRS that these are much distorted by modal polarization effects, especially at $21 / 18 \mathrm{~cm}$, and actually vary little over this band. The steep slope $(1.47 \pm 0.05)$ that results from this correlation is then apparently an artefact of these modal effects and is thus an unreliable indicator of the conal beam variations.

In order to emphasize the difficulty of interpreting such longitude-longitude correlation diagrams, we return to Fig. 4. The correlation diagram results from a 382/1375-MHz observation carried out at WSRT in 2001. We find as others have 
previously that the corresponding subpulses at the two frequencies exhibit a direct ("simultaneous") maximum correlation only of about $25 \%$. As discussed above, this associates the peaks of both profiles and leaves no direct correlation between either the leading part of the $21-\mathrm{cm}$ profile or the far trailing part of the 382-MHz one. Relevant to our discussion just above, note that the band of direct correlation containing the maximum is hardly linear and that its slope cannot be measured with very great precision-whereas the zones of indirect correlation show increasing nonlinearity. We measured the direct correlation slope to be $48^{\circ} \pm 2^{\circ}$, which corresponds to a longitude-scale ratio of $1.11 \pm 0.07$ - though the fact that the correlation maximum occurs at the same longitude is surely more significant.

Finally, well measured values of $P_{2}$ for B0809+74, in fact, exhibit only a slight frequency dependence as discussed in RRS-indeed, one which is difficult to discern in the range between about $100 \mathrm{MHz}$ and $21 \mathrm{~cm}$. It is then of interest to see what is the $P_{2}$ scaled from the $40-\mathrm{MHz}$ correlation diagram of Fig. 5. Parts of two pairs of drift bands are seen in the diagram on either side of the diagonal and closer inspection shows that these bands are not uniformly spaced. Taking a rough average of the spacing corresponding to each component, it is clear that the resulting $P_{2}$ value is some $15-17^{\circ}-$ and this probably reflects a significant increase in the emission-cone radius at $41 \mathrm{MHz}$.

\section{A geometrical emission/“absorption” model}

With the above results in mind-namely, a serviceable value for pulsar B0809+74's PA sweep rate and a fuller conception of the effects of "absorption" on its profile forms - we are in a position to construct a crude, but substantially improved model of the pulsar's emission geometry. In computing this model, we follow the course taken by DR01 for B0943+10, though in the current case "absorption" effects are seen over a much broader band. Following Kuz'min et al. (1985) and Bruk et al. (1986) we suppose that the full width of the pulsar's profile can be estimated at frequencies up to around $60 \mathrm{MHz}$ and down to some $17 \mathrm{MHz}$.

We then also suppose that much of the star's profile is observable at $18 / 21 \mathrm{~cm}$-that is, enough that power is seen from both the leading and trailing sides of the central longitude, but we have no direct way of knowing how severely "absorption" truncates this profile also. Here the star's profile has a single form, implying that our sightline traverses the outside edge of the conal beam or indeed nearly parallel to the paths of the circulating subbeams which are responsible for most of the star's emission. In short, there is a single "component" here whatever its shape, formed by the joint effects of drifting-subpulse emission, geometry and "absorption".

Basing ourselves on Popov's (1987) analysis, we take this full (un-"absorbed") half-power profile width to be $17^{\circ}$ at least-slightly larger than its strictly measured half width but consistent with a "gaussian-shaped" extrapolation of its leading edge. It is worth noticing the consistent "trapezoidal" shape of the $18 / 21-\mathrm{cm}$ profiles by contrast to the much more symmetric and Gaussian-shaped forms observed an octave higher in frequency. Therefore, Popov's indication of what the pulsar's "reconstructed" full width and shape might be (see his figure) appears to provide a plausible basis for a quantitative estimate. One the other hand, more recent analyses including Fig. 3 above suggest that the full "unabsorbed" width of B0809+74's 21-cm profile could be greater both on its leading and trailing edges. It might extend to align with both the 5and $10-\mathrm{GHz}$ profiles on its leading edge and on its trailing edge with the 382-MHz profile's trailing edge. Were this the case, we might envision an "unabsorbed" $21-\mathrm{cm}$ profile with a roughly Gaussian shape and half-power width of some $25^{\circ}$ nearly centered in Fig. 3. We will explore this range of $21-\mathrm{cm}$ profilewidth values below.

The very low frequency profile widths, however, cannot be used meaningfully without some correction for the effects of instrumental smearing. Fortunately, we have available both the PRAO 41-MHz observation in Fig. 5 for which the receiver configuation is well known as well as two new 25-MHz profiles made using the Kharkov array by Ulyanov (2004) and Popov \& Soglasnov (2004). The latter are better resolved than any previous observations and exhibit two Gaussian-shaped components with half-power widths of about $19^{\circ}$ separated by about $44^{\circ}$, making the outside $3-\mathrm{db}$ width of the profile about $63^{\circ}$. Similarly, the 41-MHz profile shows a component separation of about $30^{\circ}$ and an outside $3-\mathrm{db}$ width of $43.6 \pm 1.0^{\circ}$. Both have suffered an instrumental smearing of about $3^{\circ}$, so their corrected widths must be near $40.6^{\circ}$ and $60 .^{\circ}$ intrinsically. Using these new profiles as benchmarks, we can similarly correct the other very low frequency profile widths using the component separations and applying an estimate of the instrumental broadening.

We now have good evidence that the star's un-"absorbed" profile width above around $100 \mathrm{MHz}$ is roughly constant: the stability of $P_{2}$ (RRS) as well as the fact that longitude-longitude CCFs over wide frequency intervals peak at the same longitude. It is worth mentioning that Popov's (1986) study appears to bear this out down to $102 \mathrm{MHz}$. Moreover, we have found quantitatively that the star's steep profile-width escalation below $50 \mathrm{MHz}$ cannot be fitted unless the conal beam radius "saturates" above $1 \mathrm{GHz}$. This is equivalent to finding that B0809+74's conal beam radius behaves like those of B0329+54, 1133+16 and 2020+28, which asymptotically approach a fixed value at very high frequency when fitted with a modified Thorsett (1991) relationship (see Mitra \& Rankin's (2002) “class B" stars) - and indeed we find that B0809+74's asymptotic value is just over $5^{\circ}$ as opposed to $4.6^{\circ}$ for B1133+16, which is again about 5 times larger than the angular radius of its polar cap.

Other than this, we leave issues related to $\mathrm{B} 0809+74$ strange very high frequency behavior to future investigation. One useful line of investigation would be that of correlating pairs of well measured PSs at some 1400 and $2700 \mathrm{MHz}$. On the other hand, $\boldsymbol{S}_{\mathrm{d}}$ profiles at the highest frequencies reflect sightline traverses on the extreme outer edge of the conal beam, which may not behave in as orderly manner as the profile 
widths at lower frequencies which entail cuts across the radial maximum of the conal intensity pattern ${ }^{5}$.

With the above information and conventions established, the geometrical model in Table 2 can be computed in order to determine the magnetic latitude $\alpha$, sightline-impact angle $\beta$ and index $a$. The procedures are almost identical to those used in Deshpande \& Rankin (2001), so we will only outline them here. Four outer-cone models were computed: inner sightline traverses $(\beta<0)$ with $21-\mathrm{cm}$ "unabsorbed" profile widths of 17 and $25^{\circ}$, respectively, and a further pair for the outer $(\beta>0)$ traverse case. We reproduce only the outer pair here, however, because the resulting values are very similar in most respects. We consider only an outer cone, because this is indicated by the star's strong profile-width escalation at low frequency; as shown by Mitra \& Rankin (2002), inner cones apparently exhibit little or any variation in profile width and thus conal beam radius ${ }^{6}$. In these models, we fit only those profile widths where their full longitudinal extent can be estimated. In practice this means the profiles below frequencies of about $60 \mathrm{MHz}$ are used along with our limiting $21-\mathrm{cm}$ cases of 17 and $25^{\circ}$. For all other frequencies, we use results from the fitting to estimate the full un-"absorbed" profile widths.

The only free parameters are then the magnetic inclination $\alpha$ and the fiducial frequency $f_{0}$ as $\sin \beta=\sin \alpha / R$ and the index $a$ in the modified Thorsett (1991) relation $\rho=\rho_{0}+\left(f / f_{0}\right)^{a}$ is determined by a linear fit of $\ln \left(\rho-\rho_{0}\right)$ to $\ln \left(f / f_{0}\right)$. These two parameters are determined by minimizing $\chi^{2}$ computed from the six measured and fitted profile-width values at and below $62 \mathrm{MHz}$. Adequate fits with $\chi^{2}$ near unity can be achieved with rms errors in the profile widths of $1.0^{\circ}\left(2.0^{\circ}\right.$ at $\left.17 \mathrm{MHz}\right)$. The formal errors in $\alpha, a$, and $f_{0}$ as given in Table 2 are all smallless than half of the least significant digit. Results from the four fits are summarized in Table 3 . The outer-traverse solutions yeild $\alpha$ and $\beta$ values of around 8.5 and $+4.65^{\circ}$, respectively; whereas the inner traverse fits give values of near 9.0 and $-4.9^{\circ}$.

The full profile width curve, resulting from the above models, is then plotted in Fig. 6. Of course, as both the inner and outer traverse fits are good, this curve is indistinguishably similar for both geometries-or, said differently, the profile width behavior (as expected) gives no information about the sense of the sightline traverse. The minimum region of "absorption" (corresponding to a $21-\mathrm{cm}$ width of $17^{\circ}$ ) is shown in the figure by hatching and the larger $\left(25^{\circ}\right)$ region by cross-hatching. Overall the very low frequency width escalation is very dramatic, and Table 2 shows that this is produced by a conal beam

\footnotetext{
5 Note, for instance, that the profile widths of pulsar B0943+10 at frequencies above about $300 \mathrm{MHz}$ are not easily reconciled with those at lower frequencies where the resolved double forms and escalating width bespeak a roughly symmetrical traverse through a hollow-conal beam (DR01: Table 2). We are now learning that this overall, extremely "outer" $400-\mathrm{MHz}$ profile form reflects complex modal contributions, which appear to entail variations in both the emission height and the "absorption" (Rankin, Suleymanova \& Deshpande 2003).

${ }^{6}$ DR01's use of an inner cone geometry for pulsar B0943+10 we now see as incorrect, and we have retained this description-as it makes no difference when computing subbeam maps-for ease of comparison; see Rankin et al. (2003).
}

Table 2. B0809+74 emission geometry/“absorption" model.

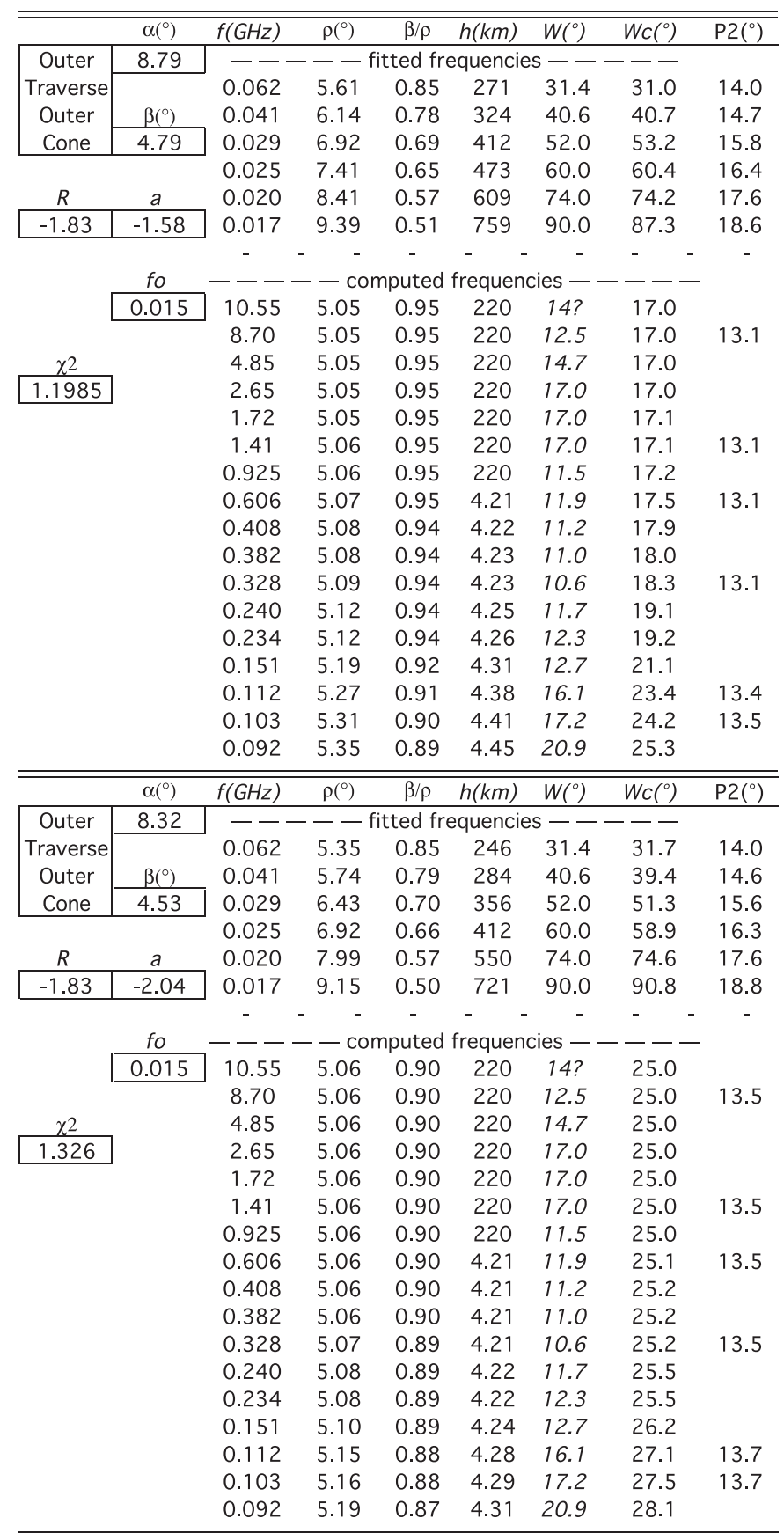

References: The fitted, relatively complete profile widths are scaled from Popov (1987), Davies et al. (1984) and Bruk et al. (1986); the "absorbed" profile widths at other frequencies were measured using Gould \& Lyne (1998), von Hoensbroech \& Xilouris (1997), Kuz'min et al. (1985, 1986, 1998), Lyne et al. (1971), Morris et al. (1981) as well as the current observations.

radius increase from just over $5^{\circ}$ to more than $9^{\circ}$. The fits can also be used to estimate the extent of the "absorption" at other frequencies. We can easily see that, for instance, the $382-\mathrm{MHz}$ profile has an estimated full width of about $18^{\circ}$ according to the minimal "absorption" fit, which can then be compared with the observed width of some $10.6^{\circ}$. Thus it would seem that the longitude of the magnetic axis must fall far on the leading edge of 
Table 3. "Absorption" model fitted values.

\begin{tabular}{ccccc}
\hline \hline $\begin{array}{c}\text { 21-cm "unabsorbed" } \\
\text { width / traverse }\end{array}$ & $\begin{array}{c}\alpha \\
\left({ }^{\circ}\right)\end{array}$ & $\begin{array}{c}\beta \\
\left(^{\circ}\right)\end{array}$ & $a$ & $\begin{array}{c}f_{0} \\
(\mathrm{GHz})\end{array}$ \\
\hline $17^{\circ}$ outer & 8.79 & 4.79 & -1.58 & 0.015 \\
$25^{\circ}$ outer & 8.32 & 4.53 & -2.04 & 0.015 \\
$17^{\circ} /$ inner & 9.13 & -4.97 & -1.71 & 0.009 \\
$25^{\circ} /$ inner & 8.97 & -4.88 & -2.16 & 0.010 \\
\hline
\end{tabular}

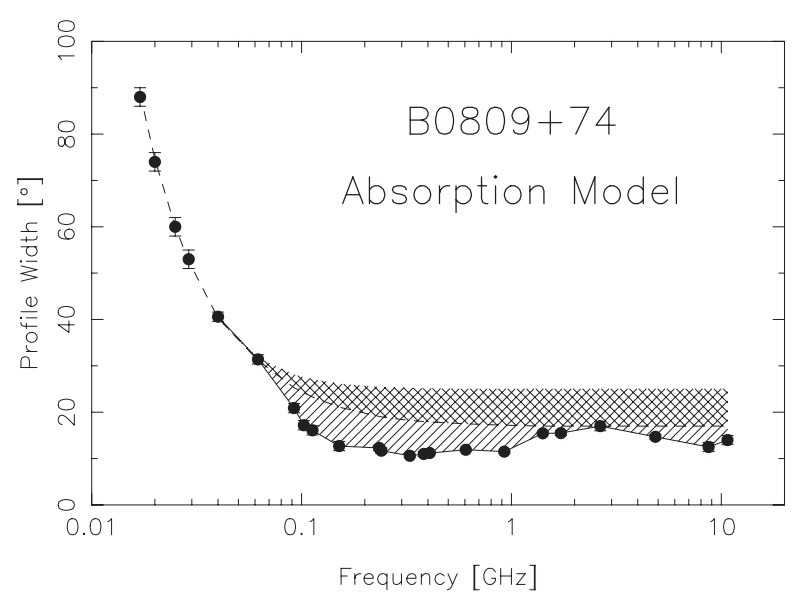

Fig. 6. Observed half-power profile widths for B0809+74 as a function of frequency showing the effects of "absorption". The symbols and error bars give the measured profile widths (for instance, of those in Fig. 2), which are then connected with a solid line. The dashed curve, by contrast, shows the full "unabsorbed" profile width according to the first "Thorsett" geometrical model in Table 2, which assumes that the $21-\mathrm{cm}$ profile suffers rather little "absorption" and thus has a full width of some $17^{\circ}$. The extent of this minimum "absorbed" region is then indicated by diagonal hatching. The larger region which could be "absorbed" is indicated by cross-hatching; here the putative $21-\mathrm{cm}$ "unabsorbed" profile width is some $25^{\circ}$ and thus large enough to encompass all of the emission features in Fig. 3. While we now know that profile-width spectra exhibit a nonlinear "Thorsett" behavior, we see here how plausible was Bartel's linear extrapolation of the highest frequency widths down to some $40 \mathrm{MHz}$; only the power-law width spectra at the very lowest frequencies (which were not then known) clearly show its falsity.

the 328-MHz profile, just above the leading half-power point. And this conclusion does not change significantly for maximal "absorption" as long as the $21-\mathrm{cm}$ profile bounds fall as in the $25^{\circ}$ model.

Well measured values of $P_{2}$ for B0809+74, in fact, exhibit only a slight frequency dependence as discussed in Rankin et al. (2005) - indeed, one which is difficult to discern in the range between about $100 \mathrm{MHz}$ and $21 \mathrm{~cm}$. Note that the model $P_{2}$ in Table 2, computed using Eq. (3) in DR01 and a 10-beam model, also exhibit a tight range of values over the above frequency interval. It would be interesting if the $P_{2}$ scaled from the $40-\mathrm{MHz}$ correlation diagram of Fig. 5 was accurate enough to test this model, but it is not.

In concluding this section it is useful to contrast the behavior of the "absorption" model in Fig. 6 with that one given two decades ago by Bartel (1981: his Fig. 1). Of course, observations over a somewhat larger band - many carried out in the mid-1980 s stimulated by Bartel's and Davies et al.'s papers - are now available to us, and the width escalation at the very lowest frequencies seen in Bruk et al. would themselves have contravened Bartel's model. An essential difference is then that the current model is based on quantitative geometrical relationships which are known to well describe the angular extent and frequency variations of most conal emission beams - i.e., the single-vector model (Radhakrishnan \& Cooke 1969; Komasaroff 1970), the outer-cone geometry at 1GHz (Rankin 1993a,b), and the modified-Thorsett-relation behavior of outer-conal beams as a function of frequency (Mitra \& Rankin 2002). In short, the current geometrical model is then necessarily non-linear in contrast to the single power-law scaling on which Bartel's "absorption" model relies.

We then see that his description fails at very low freguencies, and at the very highest frequencies it gives the impression that the narrower profiles there behave in a manner which is continuous over the entire band. We now see that this cannot be the case. Moreover, Bartel depicts the profile-width and subbeam-separation $\left(P_{2}\right)$ behaviors as if they should have the same dependence, and over the interval between about $102 \mathrm{MHz}$ and $21 \mathrm{~cm} P_{2}$ is ostensibly found to vary by nearly a factor of two. Of, course, it is exceedingly difficult to comprehend how subbeam circulation within a flaring dipole field could produce $P_{2}$ variations of this magnitude, and fortunately we now understand that the appearance of such effects is an artefact of modal polarization (Edwards \& Stappers 2003; Rankin et al. 2005).

\section{Discussion}

We have attempted in this paper to evaluate the existing best evidence pertaining to understanding B0809+74's exemplary "absorption" and then to build a broadband geometrical emission model based on this assessment. Remarkably, B0809+74 can be observed over a frequency range between more than $10 \mathrm{GHz}$ down to less than $15 \mathrm{MHz}$. Profiles over this 9-octave band are found in the published literature, including attempts to align them after having removed the dispersion. The circumstance that the star's profiles are narrower at meter wavelengths than at much higher and lower frequencies led with further study to the identification of the "absorption" phenomenon and set off a search for "superdispersion".

We now believe that these complex issues can be resolved fairly simply, and we provide a serviceable geometrical model of the pulsar's beaming geometry, based on its profile dimensions both above and below the band where the most extensive "absorption" occurs. We show that this model is in good agreement with the growth in profile scale determined by correlating the star's PSs over widely separated frequency bands as well as by computing variations in the subpulse spacing $P_{2}$.

In the course of this work we also determine a value for the star's PA sweep rate, which indeed entails studying the modal PA values at each longitude and separately estimating the slopes of each track. Not surprising - given all that we are learning about the travails of life on the outer edges of emission cones-the two slope values do not coincide. We argue, however, that the longer, steeper PA track is the most reliable geometrically and thus adopt an $R$ value of $-1.83 \pm 0.11$. 
We then model B0809+74's basic emission geometry and conclude that $\alpha$ and $|\beta|$ are some 8.5-9.0 and 4.5-4.9 ${ }^{\circ}$, respectively, as summarized in detail for the four models in Table 3. In this context we find that most of B0809+74's "absorption" occurs on the leading edge of its profiles, leaving the trailing edges mostly intact except at very high frequency. At meter wavelengths, where the "absorption" is at a maximum, the longitude of the magnetic axis appears to fall near the leading half power point of its observed profile.

Our analysis represents a marked improvement on the previous "absorption" model of Bartel (1981). It incorporates crucial profile width information at very low frequencies which were not then available. We show that apart from the "absorption" the star's profiles evolve in a manner consistent with other conal single $\left(\boldsymbol{S}_{\mathrm{d}}\right)$ stars. Our quantitative model for the angular size of the conal beam (and thus emission height) is based on the Thorsett relation and is found to agree well with the empirical measurements. The star's profile-width escalation (apart from the "absorption") as well as its spectral variations in subpulse-separation $P_{2}$ - both of which are non-linear - are now understood quantitatively as consequences of conal emission-height changes as they interact with our fixed sightline geometry.

The physical mechanism responsible for the "absorption" remains unknown, but its action in this and several other pulsars has now been delineated in considerable detail. We can thus hope that this work will provide a useful analytical basis for investigating how this mysterious "absorption" is produced.

Acknowledgements. We thank Russell Edwards and Ben Stappers for interesting and useful discussions and their assistance with some of the Westerbork observations - and in particular their generously making available to us the results of their recent polarimetry calibrations. We also thank Joeri van Leeuwen for assistance with some of the observations, Drs. Misha Popov, Volodya Soglasnov and Oleg Ulyanov for generously sharing their excellent new Kharkov observations prior to publication, and Avinash Deshpande, Misha Popov and Geoffrey Wright for other discussions. Lastly, we want to acknowledge important contributions made by our anonymous referee, whose insights and clear criticisms have materially improved the paper. Portions of this work were carried out with support from the Netherlands Organisatie voor Wetenschappelijk Onderzoek and US National Science Foundation Grants AST 99-87654 and 00-98685. This work made use of the NASA ADS astronomical data system.

\section{References}

Backer, D. C., \& Rankin, J. M. 1980, ApJS, 42, 143

Bartel, N. 1981, A\&A, 97, 384

Bartel, N., Kardeshev, N. S., Kuzmin, A. D., et al. 1981, A\&A, 93, 85 Bruk, Yu. M., Ustimenko, B. Yu., Popov, M. V., Soglasnov, V. A., \& Novikov, A. Yu. 1986, Sov. Astron. Lett., 12, 381

Cole, T. W., \& Pilkington, J. D. H. 1968, Nature, 219, 574

Davies, J. G., Lyne, A. G., Smith, F. G., et al. 1984, MNRAS, 211, 57

Deshpande, A. A., \& Rankin, J. M. 1999, ApJ, 524, 1008

Deshpande, A. A., \& Rankin, J. M. 2001, MNRAS, 322, 438
Edwards, R. T., \& Stappers, B. W. 2003, A\&A, 410, 961

Edwards, R. T., \& Stappers, B. W. 2004, A\&A, 421, 681

Gould, D. M., \& Lyne, A. G. 1998, MNRAS, 301, 253.

Hewish, A., Bell, S. J., Pilkington, J. D. H., Scott, P. F., \& Collins, R. A. 1968, Nature, 217, 709.

von Hoensbroech, A., \& Xilouris, K. M. 1997, A\&AS, 126, 121

Izvekova, V. A., Kuz'min, A. D., Malofeev, V. M., \& Shitov, Yu. P. 1990, in Pulsars, Proc. of the Lebedev Physical Institute, ed. A. D. Kuz'min, 196, 17

Kijak, J., Kramer, M., Wielebinski, R., \& Jessner, A. 1997, A\&A, 318, 63

Komesaroff, M. M. 1970, Nature, 225, 612

Kuz'min, A. D., Malofeev, V. M., Izvekova, V. A., Sieber, W., \& Wielebinski, R. 1985, Cosmic Physics Radioastronomy Laboratory, Lebedev Physical Institute, Moscow, preprint 210 (later published in a shorter form by the same authors as the paper just below)

Kuz'min, A. D., Malofeev, V. M., Izvekova, V. A., Sieber, W., \& Wielebinski, R. 1986, A\&A, 161, 183

Kuz'min, A. D., Izvekova, V. A., Shitov, Yu. P., et al. 1998, A\&AS, $127,355$.

Lyne, A. G., \& Manchester, R. N. 1988, MNRAS, 234, 519

Lyne, A. G., Smith, F. G., \& Graham, D. A. 1971, MNRAS, 153, 337.

Manchester, R. N., Taylor, J. H., \& Huguenin, G. R. 1975, ApJ, 196, 83.

Mitra, D., \& Rankin, J. M. 2002, ApJ, 577, 322 (Paper VII)

Morris, D., Graham, D. A., Sieber, W., Bartel, N., \& Thomasson, P. 1981, A\&A, 46, 421

Novikov, A. Yu., Popov, M. V., Soglasnov, V. A., Bruk, Yu. M., \& Ustimenko, B. Yu. 1984, Soviet Ast., 28, 199

Page, C. G. 1973, MNRAS, 163, 29

Pilkington, J. D. H., Hewish, A., Bell, S. J., \& Cole, T. W., 1968, Nature, 218, 126

Popov, M. V. 1987, Sov. Astron. Lett., 13, 41

Popov, M. V., \& Sieber, W. 1990, Sov. Astron., 13, 41.

Popov, M. V., \& Soglasnov, V. A. 2004, private communication

Popov, M. V., Smirnova, T. V., \& Soglasnov, V. A. 1987, Sov. Astr., 31,529

Radhakrishnan, V., \& Cooke, D. J. 1969, Ap. Lett., 3, 225

Ramachandran, R., Rankin, J. M., Stappers, B. W., Kouwenhoven, M. L. A., \& van Leeuwen, A. G. L. 2002, A\&A, 381, 993

Ramachandran, R., Backer, D. C., Rankin, J. M., Weisberg, J. M., \& Devine, K. E. 2004, ApJ, 606, 1167

Rankin, J. M. 1983, ApJ, 274, 359 (EP Paper II)

Rankin, J. M. 1993a, ApJ, 405, 285 (EP Paper VIa)

Rankin, J. M. 1993b, ApJS, 85, 145 (EP Paper VIb)

Rankin, J. M., \& Ramachandran, R. 2003, ApJ, 590, 411

Rankin, J. M., Suleymanova, S. A., \& Deshpande, A. A. 2003, MNRAS, 340, 1076

Rankin, J. M., Ramachandran, R., \& Suleymanova, S. A. 2005, A\&A, 429, 999

Shitov, Yu. P., \& Malofeev, V. M. 1985, Sov. Astr. Lett., 11, 39

Soglasnov, V. A., Smirnova, T. V., Popov, M. V., \& Kuz'min, A. D. 1981, Soviet Ast., 25, 442

Taylor, J. H., \& Huguenin, G. R. 1971, ApJ, 167, 273

Taylor, J. H., Huguenin, G. R., Hirsch, R. M., \& Manchester, R. M. 1971, ApLett, 9, 205

Thorsett, S. E. 1991, ApJ, 377, 263

Ulyanov, O. M. 2004, private communication

Vitkevich, V. V., \& Shitov, Yu. P. 1970, Nature, 225, 248 